\title{
Elevated Liver Enzymes in Patients with COVID-19: Look, but Not Too Hard
}

\author{
Andrew M. Moon ${ }^{1} \cdot$ A. Sidney Barritt IV ${ }^{1}$
}

Published online: 2 September 2020

(c) Springer Science+Business Media, LLC, part of Springer Nature 2020

Coronavirus Disease 2019 (COVID-19), due to infection with the virus termed SARS-CoV-2, has complicated the evaluation of elevated liver enzymes. Elevated liver enzymes occur in a median of $15 \%$ [1] and up to $58 \%$ [2] of patients with COVID-19. Though the most common patterns of liver enzyme abnormalities in patients with SARS-CoV-2 include elevated aminotransferases, with aspartate aminotransferase (AST) and alanine aminotransferase (ALT) typically 1-2 times the upper limit of normal [2], the prognostic significance of abnormal liver biochemistries remains uncertain. There are many potential contributing etiologies to elevated liver enzymes in patients with SARS-CoV-2 including direct liver injury, associated inflammatory responses, congestive hepatopathy, hepatic ischemia, drug-induced liver injury (DILI), and muscle breakdown [3, 4]. In one meta-analysis, an estimated $3 \%$ of patients had recognized chronic liver disease at the time of COVID-19 infection [5]. As a result, consultations for abnormal liver biochemistries in patients with COVID-19 are likely common and difficult to resolve. Clarifying a diagnosis is further complicated by the desire to limit exposure of staff assisting with or performing diagnostic testing (e.g., abdominal ultrasound or liver biopsy). In this context, there is need for more information on how best to evaluate these patients.

In the current issue of Digestive Diseases and Sciences, Bloom et al. [6] demonstrate the diagnostic difficulties of evaluating elevated liver enzymes in patients with COVID19. The authors identified twenty adult inpatients at Massachusetts General and Brigham and Women's Hospitals who were PCR-positive for SARS-CoV-2 and received inpatient hepatology consultations for abnormal liver biochemistries.

Andrew M. Moon

Andrew.Moon@unchealth.unc.edu

1 Division of Gastroenterology and Hepatology, University of North Carolina at Chapel Hill, 130 Mason Farm Road, Bioinformatics Building CB\# 7080, Chapel Hill, NC 27599-7080, USA
Laboratory and clinical data were retrospectively reviewed by three senior hepatologists who assigned a rank-order list of the top three potential etiologies, providing recommendations for additional evaluations. Patients with COVID-19 in this study were middle-aged (median 46 years), $90 \%$ male, $55 \%$ Hispanic, and $40 \%$ had underlying chronic liver disease. Most had a hepatocellular (64\%) or cholestatic (29\%) pattern of liver biochemistries. Blood chemistries reflective of liver synthetic function were generally normal. The most common diagnoses for these patients were COVID-related liver injury and DILI, but agreement on the most likely diagnosis was low among the three hepatologists and the original consultant ( $\kappa$ agreement 0.10 ). Conversely, all were in general agreement with the diagnostic work-up, which included liver enzyme monitoring for all patients. Abdominal ultrasound was recommended for a minority and was often discouraged. Similarly, cross-sectional imaging and liver biopsy were not recommended for any patients.

This investigation of the diagnosis and work-up of elevated liver enzymes in patients with COVID-19 provides a timely glimpse into the difficulties encountered caring for these patients. However, study results should be interpreted in the context of potential limitations. This study included a small patient sample, and results may lack generalizability to other settings given that it was conducted at a large academic medical center early in the US COVID-19 epidemic (March-April 2020), when understanding of the hepatic effects of SARS-CoV-2 was limited. Furthermore, restricting this cohort to patients with a hepatology consultation may have selected for more complex patients, which could account for the large proportion of included patients with chronic liver disease. Lastly, without follow-up data in these patients, it is impossible to evaluate the accuracy of the ranked diagnoses.

These potential limitations do not diminish the immediate clinical implications of this study. These results underscore the immense diagnostic challenge of elevated liver enzymes in COVID-19, given the large degree of disagreement among 
senior hepatologists at a tertiary academic medical center. The majority of COVID-19 patients receive care at nonacademic medical centers without access to hepatology consultation. Therefore, guidance on a streamlined approach to evaluate these patients is essential.

The current consensus statements from GI and liver societies endorse a thorough evaluation for alternative etiologies of elevated liver enzymes in patients with COVID-19 and consideration of liver enzyme monitoring during hospitalization $[1,2]$. There is no current recommendation for or against liver biopsy for patients with COVID-19 who develop acute liver injury. For the time being, however, liver biopsy should rarely be used for the evaluation of abnormal liver biochemistries in patients with COVID-19 for a number of reasons:

First, no specific histopathological findings in patients with COVID-19 have emerged [3] and many centers may not have an experienced liver pathologist. There is one report of possible coronavirus particles in hepatocyte cytoplasm [7], which may suggest direct viral infection, but it is possible that these findings are due to autolysis rather than direct viral inclusions [8]. Given the lack of histopathological findings specific to COVID-related liver injury, the primary benefit of biopsy would be to rule out alternative etiologies of liver injury, which can generally be evaluated without the need for liver biopsy.

Second, liver biopsy is unlikely to lead to a change in management or provide prognostic information. In this study by Bloom et al., there was broad agreement among included hepatologists regarding diagnostic evaluation, suggesting that the diagnosis did not have a strong bearing on follow-up recommendations. Furthermore, there have been inconsistent findings regarding the prognostic importance of elevated liver enzymes in COVID-19 [9]. It is likely that well-established risk factors, such as age and medical comorbidities (including cirrhosis), are more important predictors of COVID-19 outcomes than are liver biochemistries. Moreover, in a recent large cohort study of hospitalized patients with COVID-19, the prevalence of severe acute liver injury was exceedingly low [10].

Third, the potential risks of liver biopsy for COVID-19 will extend to both patient and staff. Procedural risks of liver biopsy include hemorrhagic complications, biliary injury, pneumothorax, and even death. For patients with COVID-19, there are added risks of SARS-CoV-2 transmission to ancillary staff members involved in patient transportation or in procedure areas where biopsies are performed. For the vast majority of patients, these risks will likely outweigh its uncertain benefits.
In conclusion, this study by Bloom et al. reports that, early in the COVID-19 pandemic, there was significant disagreement among senior hepatologists with regard to the cause of abnormal liver biochemistries in a small group of SARS-CoV-2 infected patients, but consistency with regard to follow-up recommendations. As demonstrated in this study, a diagnostic approach for elevated liver enzymes in COVID-19 should include: (1) thorough evaluation of alcohol and medication use, (2) laboratory assessment of liver synthetic function and portal hypertension (i.e., platelet count, albumin, PT/INR), (3) serological and virological testing in order to exclude common prevalent liver diseases such as viral hepatitis, (4) ongoing monitoring of liver enzymes, (5) imaging reserved for patients with high pre-test probability of biliary obstruction or thrombosis, and (6) liver biopsy in rare cases when results may change care (e.g., to assess for rejection in a post-liver transplant patient). We applaud ongoing efforts to provide guidance on the management of patients with COVID-19, given that best practices will evolve along with our understanding of SARS-CoV-2.

Author's contribution All authors approved the final version of this manuscript. Andrew M. Moon is the guarantor of this paper and contributed to study concept, drafting, and critical revision of manuscript. A. Sidney Barritt contributed to study concept, critical revision of manuscript.

Funding This research was supported in part by NIH Grant T32 DK007634.

\section{Compliance with Ethical Standards}

Conflict of interest No disclosures or conflict of interest.

\section{References}

1. Sultan S, Altayar O, Siddique SM, et al. AGA Institute rapid review of the gastrointestinal and liver manifestations of COVID19 , meta-analysis of international data, and recommendations for the consultative management of patients with COVID-19. Gastroenterology. 2020;159:320-334.

2. Clinical best practice advice for hepatology and liver transplant providers during the COVID-19 Pandemic: AASLD expert panel consensus statement, 2020. Available at: https://www.aasld.org/ sites/default/files/2020-06/AASLD-COVID19-ExpertPanelCons ensusStatement-June252020-v2-FINAL.pdf. Accessed August 4, 2020.

3. Jothimani D, Venugopal R, Abedin MF, Kaliamoorthy I, Rela M. COVID-19 and liver. J Hepatol. 2020. https://doi.org/10.1016/j. jhep.2020.06.006.

4. Schaefer EAK, Arvind A, Bloom PP, Chung RT. Interrelationship between coronavirus infection and liver disease. Clin Liver Dis (Hoboken). 2020;15:175-180. 
5. Mantovani A, Beatrice G, Dalbeni A. Coronavirus disease 2019 and prevalence of chronic liver disease: A meta-analysis. Liver Int. 2020;40:1316-1320.

6. Bloom PP, Pasricha T, Andersson KL, et al. Hepatology consultants often disagree on etiology of abnormal liver biochemistries in COVID-19 but agree on management. Dig Dis Sci. (Epub ahead of print). https://doi.org/10.1007/s10620-020-06495-w.

7. Wang Y, Liu S, Liu H, et al. SARS-CoV-2 infection of the liver directly contributes to hepatic impairment in patients with COVID-19. J Hepatol. 2020. https://doi.org/10.1016/j. jhep.2020.05.002.

8. Bangash MN, Patel JM, Parekh D, et al. SARS-CoV-2: is the liver merely a bystander to severe disease? J Hepatol. 2020. https://doi. org/10.1016/j.jhep.2020.05.035.
9. Kovalic AJ, Huang G, Thuluvath PJ, Satapathy SK. Elevated liver biochemistries in hospitalized Chinese patients with severe COVID-19: systematic review and meta-analysis. Hepatology. 2020. https://doi.org/10.1002/hep.31472.

10. Elmunzer BJ, Spitzer RL, Foster LD, et al. Digestive manifestations in patients hospitalized with COVID-19. medRxiv. 2020. https://doi.org/10.1101/2020.07.07.20143024v2.

Publisher's Note Springer Nature remains neutral with regard to jurisdictional claims in published maps and institutional affiliations. 